\title{
PERCEPCIÓN AFECTIVA DEL ALUMNADO EN EDUCACIÓN FÍSICA PRIMARIA EN LA PROVINCIA DE ALICANTE
}

\section{AFFECTIVE PERCEPTION OF STUDENTS IN PRIMARY PHYSICAL EDUCATION IN THE PROVINCE OF ALICANTE}

\author{
${ }^{1}$ Andrea Jordá-Espi, ${ }^{1}$ Pedro Gil-Madrona, ${ }^{2}$ Felipe Nicolás Mujica Johnson, ${ }^{1}$ Lorena Pascual-Frances \\ ${ }^{1}$ Universidad de Castilla La Mancha, España 2 Universidad Católica de Temuco, Chile \\ Envío original: 2018-10-27. Reenviado: 2019-04-04. Aceptado: 2019-04-04. Publicado: 2019-06-10
}

\section{RESUMEN}

La interacción motriz, es un aspecto que determina el tipo de juego sociomotor, aportando características específicas que diferencian a este didáctico recurso de la clase de educación física. Este estudio tiene como propósito identificar la percepción afectiva del alumnado de $5^{\circ}$ y 6 ํㅜ curso de educación primaria en los juegos de cooperación y de cooperación-oposición, en la clase de educación física, en función del sexo. El estudio es de tipo descriptivo y sus participantes son 70 estudiantes pertenecientes a la provincia de Alicante, con edades comprendidas entre los 10 y 12 años. Los datos fueron recopilados por medio de la encuesta PANAS. Los principales resultados indican que en los juegos de cooperación se percibe un menor afecto positivo que en los de cooperación-oposición. Se concluye que el afecto positivo predomina en ambos juegos sociomotrices, por lo que puede ser un contenido ideal para construir un buen ambiente de aprendizaje. Según el sexo, se concluye que las mujeres perciben más afecto positivo que los hombres en los juegos de cooperación-oposición y menos en los juegos de cooperación sin oposición. En cuanto al afecto negativo, en ambos juegos los hombres lo perciben más que las mujeres.

Palabras claves: emociones, competición, bienestar subjetivo, socialización, juego motor.

\begin{abstract}
The motor interaction is an aspect that determines the type of sociomotor game, providing specific characteristics that differentiate this didactic resource from the physical education class. The purpose of this study is to identify the affective perception of the 5th and 6th grade students of primary education in the games of cooperation and cooperation-opposition, in the physical education class, according to the sex and the country of origin of the students. The study is descriptive and its participants are 70 students belonging to the province of Alicante, aged between 10 and 12 years. The data was collected through the PANAS survey. The main results indicate that in cooperative games less positive affect is perceived than in cooperationopposition. It is concluded that positive affect predominates in both sociomotor games, so it can be an ideal content to build a good learning environment. According to sex, it is concluded that women perceive more positive affection than men in cooperation-opposition games and less in unopposed cooperation games. Regarding negative affect, in both games men perceive it more than women.
\end{abstract}

Keywords: emotions, competition, subjective well-being, socialization, motor game. 
El juego motor se enmarca en la ludomotricidad, que alude al placer por el juego, al deseo de una actividad entretenida (Parlebas, 2001), por lo que durante el desarrollo de aquella actividad motriz lúdica, el afecto tiene una posición relevante. El aspecto lúdico, permite resaltar el afecto positivo como uno de sus objetivos, ya que responde a una capacidad humana de gozar el ocio en el tiempo presente (Trigo y De la Piñera, 2000). El juego motor, es practicado con una finalidad educativa en la clase de Educación Física (EF), por lo que en el presente estudio será analizado desde la dimensión afectiva. Existe una clasificación del juego motor de acuerdo a su tarea motriz y se pueden dividir según el tipo de comunicación motriz, en psicomotrices, sociomotrices de cooperación, sociomotrices de oposición y sociomotrices de cooperación-oposición (Serna, Muñoz, Lavega, March-Llanes, Sáez de Ocáriz y Hileno, 2017).

En esta investigación se abordarán dos tipos de interacción motriz, que son los de cooperación y los de cooperación-oposición. El juego motor cooperativo, mantiene una lógica interna de comunicación motriz, donde los practicantes son siempre compañeros y la tarea es generar una producción motriz colectiva, mientras que el juego motor de cooperaciónoposición, además de tener en su lógica interna la comunicación motriz, se le agrega la contracomunicación motriz, en donde los practicantes tienen que lograr contrarrestar la realización de la tarea de los otros jugadores, transformándose en adversarios (Parlebas, 2001). La diferente lógica interna que presentan estos juegos motores, ha motivado el estudio de la calidad que tiene la interacción social que se produce, identificando que los alumnos exhiben un mayor número de interacciones sociales positivas, como son compartir, ayudar, o proteger, en juegos estructurados de manera cooperativa que en aquellos otros de tipo competitivo (Contreras y García, 2011).

\section{Afectividad en la Educación Física}

Desde la perspectiva de la motricidad humana, la dimensión afectiva en la EF es valiosa por sí misma, porque entiende al cuerpo en su globalidad, no solo como un conjunto de músculo, huesos, articulaciones y su conexión biomecánica, sino más bien como un cuerpo que piensa, que siente, que escucha, que comunica, que expresa (Pellicer, 2011). En este sentido, el movimiento humano es comprendido como un acto lleno de afecto, sentido, significancia, cultura y experiencia, ya que "no es posible distinguir o separar la intención del acto pues la primera ya es una acción que se genera desde las matrices constitutivas del ser biológico en despliegue como ser cultural" (Toro, 2006, p. 68). Por lo mismo, la afectividad ha de ser estudiada en cualquiera de los contextos educativos, para de esta forma conocer, comprender e interpretar el escenario pedagógico.

Uno de los componentes más importantes de la afectividad es la emoción, por lo que "uno de los grandes logros de la EF, sería que el alumnado durante las actividades motrices, aprenda a construir emociones positivas para su bienestar subjetivo enmarcadas en la ética" (Mujica, 2018, p. 70). Por ejemplo, el alumnado puede experimentar emociones placenteras en la asignatura de EF, señalando que se sienten tranquilos (algo, bastante o muy tranquilos) (Gil y Martínez, 2016). En la actualidad se puede observar un aumento de las investigaciones científicas por este tema, incluyendo el ámbito de la actividad física y el deporte, lo cual favorece el debate de la formación docente y proporciona un conocimiento teórico-práctico al 
profesorado, que es beneficioso para quienes se propongan favorecer un buen clima de aprendizaje. Como estrategia, el juego motor ha sido reconocido como una actividad propicia para favorecer el bienestar subjetivo (BS) y con mayor énfasis, el de carácter sociomotriz sin competición, suscitando mayor cantidad de estas emociones positivas para el BS (Mujica, Orellana y Concha, 2017).

No obstante, algunos autores señalan que la asignatura de EF es una de las últimas materias que ha abordado el tema de la educación moral en sus programaciones curriculares (Hernández-Mendo y Planchuelo, 2014). Algunos estudios que han abordado las actitudes y los valores en la EF escolar de España, han evidenciado que el alumnado valora la innovación y el dinamismo de las actividades motrices, como el interés del profesorado por lo que ellos sienten durante las clases (Gutiérrez y Pilsa, 2006), por otra parte han manifestado un positivo interés por la posibilidad de construir hábitos de vida saludable y valoran de buena forma las adecuadas relaciones sociales que se producen entre pares y con el docente (Gaviria y Castejón, 2016). Es importante señalar que en la clase de EF existe un alumnado con características muy diversas, por lo que es fundamental pensar los contenidos de la asignatura desde una perspectiva inclusiva, más aún, cuando recientes estudios han visibilizado desigualdades de género en la EF y la práctica deportiva (González-Palomares, Táboas-Pais y Rey-Cao, 2017; Lamoneda y Huertas, 2017; Abellán, Sáez-Gallego y Reina, 2018).

Con la intención de contribuir al conocimiento sobre la afectividad en el ámbito de la asignatura, el objeto de estudio es identificar la percepción afectiva del alumnado en la asignatura de educación física en los juegos de cooperación y de cooperación-oposición, en función del sexo.

\section{MÉTODOLOGÍA}

Para responder al objetivo de investigación, se ha optado por un enfoque cuantitativo, con un diseño no experimental y de tipo descriptivo.

Participantes. En el estudio participaron un total de 70 estudiantes (35 hombres y 35 mujeres) de $5^{\circ}$ y $6^{\circ}$ curso de educación primaria, con edades comprendidas entre los 10 y 12 años, pertenecientes a un centro educativo público situado en la provincia de Alicante.

Instrumento y análisis de datos. Para alcanzar el objetivo del estudio, se empleó la encuesta de valoración de aspectos positivos y negativos en las clases de EF de la versión PANAS ("Positive and Negative Affect Schedule"), validada originalmente por Watson, Clark y Tellegen (1988) con estudiantes universitarios de psicología, reportando una fiabilidad aceptable, con un coeficiente alfa de Cronbach entre .86 a .90 para afecto positivo (AP) y entre .84 a .87 para afecto negativo (AN). Respecto a su validez convergente, los análisis presentan correlaciones que oscilan entre .89 a .95 , mientras que su validez discriminante presenta correlaciones que van desde -.02 a -.18 (Watson et al., 1988). Esta escala fue posteriormente validada en castellano por Sandín (2003) para niños/as y adolescentes, específicamente en una muestra española de la Comunidad de Navarra, reportando una fiabilidad aceptable, al igual que una adecuada validez convergente y discriminante (entre .91 y .77 para el caso de la sub-escala PANAS positivo y de.85 y 0.82 en negativos). En término más recientes, esta 
misma escala fue validada por González y Valdez (2015) en una muestra de niños y adolescentes de México, reportando una fiabilidad aceptable, una estructura bidimensional estable de afecto positivo-negativo, así como evidencia que confirma la validez porque los reactivos de AP se separan adecuadamente de los de AN y finalmente, una validez convergente de la escala con una medida de resiliencia. Esta encuesta se compone de 20 ítems y presenta una estructura bidimensional: AP y AN, con 10 ítems para cada sub-escala (entre .86 a .90 para la escala del Afecto Positivo; y de .84 a .87 para la del Afecto Negativo).

El análisis estadístico realizado es de tipo descriptivo. La técnica de estadística que se utilizó es "la distribución de frecuencia relativa acumulada o frecuencia acumulada en porcentajes, que es la frecuencia acumulada dividida entre la frecuencia total" (Spiegel y Stephens, 2001, p. 39).

Procedimiento. La primera acción fue tomar contacto con el equipo directivo del establecimiento educacional y solicitar el permiso para llevar a cabo el estudio. En segunda instancia fue comunicarse con la maestra de Educación Física que imparte clases en el $5^{\circ}$ y $6^{\circ}$ curso de Primaria, a la cual se le informó el proyecto de investigación, que cumple con procedimientos acordes a la Declaración de Helsinki, y se le solicitó su colaboración. Por último, se dio lugar a solicitar el consentimiento informado de los estudiantes y de sus padres y/o tutores, en el que se les informó del carácter voluntario de participación, además de la confidencialidad y anonimato de los datos. Lo siguiente fue participar de dos sesiones de EF en cada curso, las cuales constan de 45 minutos de duración y que, en función del sexo, cuentan con una participación mixta del alumnado.

En la primera sesión de cada curso la maestra de EF, gestionó los siguientes cuatro juegos motores de cooperación sin oposición: a) la espiral; b) cruzar el lago; c) Bancooperación; y d) con los zancos y a lo loco. Mientras que, en la segunda sesión de cada curso, la maestra de EF gestionó los siguientes cuatro juegos motores de cooperaciónoposición: a) lucha suiza; b) 10 pases; c) fútbol sin balón; y d) barrer la casa. Una vez concluida cada sesión, se aplicó al alumnado de manera individual la encuesta PANAS.

\section{RESULTADOS}

El resultado general de los afectos en los juegos sociomotores, se presenta en la Tabla 1 , donde se puede observar que los AP son los más percibidos y los AN son los menos percibidos. Las diferencias encontradas en el AP de los juegos sociomotores según su interacción motriz, son que el alumnado en los de cooperación sin oposición lo percibe en menos cantidad en las categorías muchas veces y nunca, pero en mayor cantidad en la categoría pocas veces. Lo mismo sucede con la percepción del AN, ya que los juegos sociomotores de cooperación sin oposición, en percibido en menor cantidad en la categoría muchas veces y nunca, pero en mayor cantidad en la categoría pocas veces. 
Tabla 1.

Distribución en porcentajes de la percepción de los afectos positivos y negativos en los juegos sociomotores de cooperación sin oposición y de cooperación-oposición.

\begin{tabular}{ccccc}
\hline Frecuencia & AP JSMCSO & AP JSMCO & AN JSMCSO & AN JSMCO \\
\hline Muchas veces & 54.86 & 56.72 & 2.71 & 4 \\
Pocas veces & 39 & 35.71 & 27.72 & 17.86 \\
Nunca & 6.14 & 7.57 & 69.57 & 78.14 \\
\hline
\end{tabular}

Nota: JSMCSO: Juego sociomotor de cooperación sin oposición; JSMCO: Juego sociomotor de cooperación-oposición. Fuente: elaboración propia.

Los elementos de la percepción afectiva en el juego sociomotor de cooperación sin oposición, se pueden apreciar en la Tabla 2. Los referidos al AP percibido en mayor cantidad, son la vitalidad, la actividad y el interés. En cuanto a los elementos del AN percibido en mayor cantidad, se encuentra el nerviosismo, la intranquilidad y la vergüenza.

Tabla 2.

Distribución en porcentajes de la intensidad de los elementos afectivos en los juegos motores de cooperación sin oposición.

\begin{tabular}{cccc}
\hline Elemento afectivo & Nunca & $\begin{array}{c}\text { Algunas } \\
\text { veces }\end{array}$ & $\begin{array}{c}\text { Muchas } \\
\text { veces }\end{array}$ \\
Siento miedo & & 17.14 & 0 \\
Soy un/a chico/a activo/a & 82.86 & 15.71 & 81.43 \\
Siento sensaciones corporales de estar & 61.43 & 31.43 & 7.14 \\
intranquilo/a & & & \\
Soy una persona atenta, esmerada & 1.43 & 40 & 58.57 \\
Soy un/a chico/a decidido/a & 8.58 & 45.71 & 45.71 \\
Me siento nervioso & 58.57 & 32.86 & 8.57 \\
Me siento inspirado/a & 8.57 & 48.57 & 42.86 \\
Soy vergonzoso/a & 42.86 & 52.86 & 4.28 \\
Soy un/a chico/a despierto/a, despabilado/a & 7.14 & 55.72 & 37.14 \\
Tengo mal humor (me altero o irrito) & 75.71 & 22.86 & 1.43 \\
Me siento orgulloso/a (de algo), satisfecho/a & 1.43 & 38.57 & 60 \\
Me entusiasmo (por cosas, personas, etc.) & 17.14 & 50 & 32.86 \\
Estoy enfadado/a furioso/a & 72.86 & 27.14 & 0 \\
Soy un/a chico/a asustadizo/a & 80 & 20 & 0 \\
Me siento culpable & 77.14 & 20 & 2.86 \\
Siento que tengo vitalidad, energía & 4.29 & 20 & 75.71 \\
Me siento disgustado/a o molesto/a & 74.28 & 22.86 & 2.86 \\
Soy una persona animada, suelo emocionarme & 8.57 & 40 & 51.43 \\
Me siento tenso/a, agobiado/a, con sensación de & 70 & 30 & 0 \\
estrés & & & \\
Me intereso por la gente o las cosas & 1.43 & 35.71 & 62.86 \\
\hline Fuen & & &
\end{tabular}

Fuente: elaboración propia. 
En cuanto a la diferencia por sexo de la percepción afectiva en los juegos sociomotores de cooperación sin oposición, se puede apreciar en la Tabla 3, que los chicos perciben en mayor cantidad el AP y el AN.

Tabla 3.

Distribución en porcentajes de la percepción afectiva según el sexo en los juegos sociomotores de cooperación sin oposición.

\begin{tabular}{ccccc}
\hline Frecuencia & AP (chicos) & AP (chicas) & AN (chicos) & AN (chicas) \\
\hline Muchas veces & 56.57 & 53.14 & 4 & 1.43 \\
Pocas Veces & 37.71 & 40.29 & 31.71 & 23.71 \\
Nunca & 5.72 & 6.57 & 64.29 & 74.86 \\
\hline
\end{tabular}

Fuente: elaboración propia.

Los elementos de la percepción afectiva en el juego sociomotor de cooperaciónoposición, se pueden apreciar en la Tabla 4. Los referidos al AP que fueron percibidos en mayor cantidad, son la actividad, la vitalidad y el interés hacia el entorno. En cuanto a los elementos del AN percibido en mayor cantidad, se encuentra el nerviosismo, la vergüenza y la intranquilidad.

Tabla 4.

Distribución en porcentajes de la intensidad de los elementos afectivos en los juegos motores de cooperación-oposición.

\begin{tabular}{cccc}
\hline Elemento afectivo & Nunca & $\begin{array}{c}\text { Algunas } \\
\text { veces }\end{array}$ & Muchas veces \\
Siento miedo & 81.43 & 15.71 & 2.86 \\
Soy un/a chico/a activo/a & 0 & 25.71 & 74.29 \\
Siento sensaciones corporales de estar & 72.86 & 21.43 & 5.71 \\
intranquilo/a & & & \\
Soy una persona atenta, esmerada & 1.43 & 37.14 & 61.43 \\
Soy un/a chico/a decidido/a & 7.14 & 45.72 & 47.14 \\
Me siento nervioso & 64.29 & 25.71 & 10 \\
Me siento inspirado/a & 22.86 & 42.86 & 34.28 \\
Soy vergonzoso/a & 64.29 & 27.14 & 8.57 \\
Soy un/a chico/a despierto/a, & 7.14 & 31.43 & 61.43 \\
despabilado/a & & & \\
Tengo mal humor (me altero o irrito) & 78.57 & 18.57 & 2.86 \\
Me siento orgulloso/a (de algo), & 5.71 & 35.72 & 58.57 \\
satisfecho/a & & & \\
Me entusiasmo (por cosas, personas, & 21.43 & 38.57 & 40 \\
$\quad$ etc.) & & & \\
Estoy enfadado/a furioso/a & 88.57 & 10 & 1.43 \\
Soy un/a chico/a asustadizo/a & 82.86 & 15.71 & 1.43 \\
Me siento culpable & 81.43 & 17.14 & 1.43 \\
Siento que tengo vitalidad, energía & 0 & 27.14 & 72.86
\end{tabular}




\begin{tabular}{|c|c|c|c|}
\hline Me siento disgustado/a o molesto/a & 88.57 & 10 & 1.43 \\
\hline $\begin{array}{l}\text { Soy una persona animada, suelo } \\
\text { emocionarme }\end{array}$ & 8.57 & 44.29 & 47.14 \\
\hline $\begin{array}{l}\text { Me siento tenso/a, agobiado/a, con } \\
\text { sensación de estrés }\end{array}$ & 78.57 & 17.14 & 4.29 \\
\hline Me intereso por la gente o las cosas & 1.43 & 28.57 & 70 \\
\hline
\end{tabular}

Fuente: elaboración propia.

En cuanto a la diferencia por sexo de la percepción afectiva en los juegos sociomotores de cooperación-oposición, se puede apreciar en la Tabla 5, que las chicas perciben en mayor cantidad el AP y los chicos perciben en mayor cantidad el AN.

Tabla 5.

Distribución en porcentajes de la percepción afectiva según el sexo en los juegos sociomotores de cooperación-oposición.

\begin{tabular}{ccccc}
\hline Frecuencia & AP (chicos) & AP (chicas) & AN (chicos) & AN (chicas) \\
\hline Muchas veces & 55.43 & 58 & 5.71 & 2.28 \\
Pocas Veces & 35.71 & 35.71 & 20.86 & 14.86 \\
Nunca & 8.86 & 6.29 & 73.43 & 82.86 \\
\hline
\end{tabular}

Fuente: elaboración propia.

\section{DISCUSIÓN}

La percepción de AP por sobre el AN en los juegos sociomotores, es un hallazgo que ha sido expuesto por otros estudios (Lavega, Filella, Agulló, Soldevila y March, 2011; Sáez de Ocáriz, Lavega, Mateu y Rovira, 2014; Duran, Lavega, Salas, Tamarit y Invernó, 2014; Medina, 2015; Caballero, Alcaraz, Alonso y Yuste, 2016), demostrando la importancia que tiene el juego sociomotor en el BS del alumnado, convirtiéndose en un recurso pedagógico en la clase de EF. La alta percepción del AP en los juegos de cooperación-oposición, es un resultado que difiere de otros estudios que concluyen lo contrario, señalando que "los juegos de cooperación sin competición son aquellos que obtienen los mejores resultados para desencadenar emociones positivas a los alumnos" (Miralles, Filella y Lavega, 2017, p. 92). El factor del resultado del juego es muy influyente en esta tendencia, ya que el juego cooperativo sin victoria "activa emociones positivas y ambiguas, y al no haber resultado esas emociones ambiguas actúan como positivas" (Molina, 2016, p. 128). Así, algunos estudios han llegado a concluir que la percepción con mayor intensidad de emociones positivas para el BS se asocia a los juegos de los dominios sociomotores y más concretamente en aquellos en los que el resultado no es tomado en consideración (Duran et al., 2014). Sin embargo, en este estudio se ha identificado una alta presencia de AP en los juegos cooperativos y de oposición.

Los hombres, manifiestan percibir mayor AP que las mujeres en los juegos de cooperación sin oposición, mientras que las mujeres perciben mayor AP en los juegos de cooperación-oposición. Que ellas perciban mayor cantidad de AP en los juegos de competición, 
puede ser explicado por el significado que le otorgan al juego, como ha sido identificado en un estudio cualitativo de la vivencia emocional en EF Primaria, el cual indica que las niñas atribuyeron la vivencia de emociones positivas al bienestar que origina la colaboración con otras personas, el intercambio de risas o jugar con una amiga, a diferencia de los niños que olvidan estos aspectos (Alcaraz-Muñoz, Alonso y Yuste, 2017). No obstante, también se ha reconocido AN por parte del género femenino y coincide con que en otros estudios se ha identificado que la competencia puede excluir a las mujeres y tornar violento el juego sociomotriz (Gaviria y Castejón, 2016). En cuanto al AN, los hombres en ambos juegos lo han percibido más que las mujeres, sin embargo, se debe considerar que los datos fueron tratados con una estadística descriptiva, de modo que en otro estudio que ha analizado esta variable con una estadística inferencial, ha determinado no identificar diferencias significativas en la variable de sexo (Miralles et al., 2017).

La percepción de AP, relacionada a sentirse una persona activa, atenta y con interés por el entorno, obtuvo la mayor presencia en los dos tipos de juego sociomotor, por lo que por medio de la práctica de estos juegos, el alumnado identifica características personales de un buen autoestima, lo cual se ha relacionado a la meta del aprendizaje cooperativo, debido a que el hecho de que todo el alumnado en la clase tenga que enfrentarse a un reto común, favorecerá el trabajo cooperativo, comenzando a desarrollar así su autoestima a través de la dimensión social (García, 2011). Además, la predominancia del AP en los juegos sociomotores estudiados, se relaciona con un espacio inclusivo, donde la mayor parte del alumnado puede participar activamente, lo cual ha sido también identificado en un estudio similar, el cual concluye que todos los/as escolares "fueron capaces de participar activamente en los juegos de cooperación, protagonizando mayoritariamente conductas motrices cooperativas ajustadas" (Lavega, Planas y Ruiz, 2014, p. 48).

La mayor percepción de AN durante los juegos de cooperación-oposición, ha sido explicada por la competencia, debido a que las emociones negativas serían suscitadas por la derrota (Miralles et al., 2017), lo cual se acrecienta en la medida que el alumnado tengan un gran deseo de conseguir la victoria. Obtener la victoria de un juego, es un hecho que puede desencadenar emociones positivas en el alumnado, lo cual es explicado desde la teoría de memoria del yo. Esta teoría indica que dependiendo de la discrepancia entre la meta actual y la meta ideal que se tiene como expectativa de logro, se producirán emociones de un valor u otro, porque "las emociones positivas reflejan una tasa aceptable de reducción en la discrepancia, mientras que las emociones negativas reflejan una falla en la reducción de discrepancias" (Conway y Pleydell-Pearce, 2000, p. 269). Así, ganar un juego de carácter competitivo, supondría una gran satisfacción para el escolar, así como un gran refuerzo positivo para su autoestima (García, 2011). Considerando las respuestas emocionales del alumnado, el deporte competitivo genera importantes situaciones de aprendizajes en la asignatura, los cuales pueden ser extrapolados a los desafíos que vivencia un ciudadano en su vida diaria, entendiendo que la competencia se encuentra en la base de un sistema democrático, donde dos o más personas se ven enfrentadas para conseguir un mismo objetivo, ya sea a nivel educacional, laboral o político, por lo que aprender a competir se convierte en un desafío moral.

Los afectos negativos que fueron percibidos en mayor cantidad, son el nerviosismo, la intranquilidad y la vergüenza, emociones que afectan el BS del alumnado durante su práctica ludomotriz. La vergüenza, ha sido atribuida en otros estudios desarrollados en esta asignatura 
a la dificultad de la tarea, a las burlas y al pensamiento pesimista (Mujica, Orellana, Aránguiz y González, 2016), a la competencia (Sáez, Lavega, Lagardera, Costes y Serna, 2014) y a las actividades individuales con exposición social (Rodríguez y Rocu, 2016). El nerviosismo, ha sido atribuido a la falta de experiencia motriz, a la inseguridad de logro de una meta, al pensamiento pesimista y al temor a la evaluación, mientras que la intranquilidad, ha sido atribuida a la ausencia de logro de superación en la tarea motriz y al pensamiento de inseguridad de logro (Mujica et al., 2016). También esta última emoción ha sido atribuida por el alumnado al impacto fuerte de un balón en el aprendizaje de voleibol (Canales y Pina, 2014; Monforte y Pérez-Samaniego, 2017), a la competición (Duran, et al., 2014), a la insatisfacción corporal y a las críticas o burlas en relación con la apariencia física (Camacho y Aragón, 2014). Los diferentes estudios mencionados que han abordado las emociones negativas en los juegos sociomotrices, indican varios factores que pueden haber incidido en este tipo de afecto, por lo que sería de gran utilidad que se realicen estudios de corte cualitativo, que profundicen en la dimensión afectiva durante los juegos sociomotores de cooperación y cooperación-oposición.

\section{CONCLUSIONES}

En relación al objetivo del estudio, se identificó que el AP predomina en el juego sociomotor de cooperación sin oposición y de cooperación-oposición. De acuerdo al análisis en función del sexo del alumnado, las mujeres perciben más AP que los hombres en el juego sociomotor de cooperación-oposición y menos que los hombres en el juego sociomotor de cooperación sin oposición. En cuanto al AN, en ambos juegos sociomotores los hombres lo perciben más que las mujeres.

La información recogida podría ayudar en el futuro a elaborar diseños de intervención para favorecer el BS en las experiencias afectivas durante las clases de EF, mejorar el ambiente de aprendizaje, al igual que la autoestima y la adherencia a la práctica de actividades físico-deportivas. No obstante, se hace necesaria la realización de otras investigaciones que aborden las variables estudiadas, junto a otras variables y contextos del ámbito educativo, las cuales pueden ser abordadas con diferentes enfoques y diseños investigativos, para así poder ampliar la perspectiva y generar una perspectiva comprensiva de la dimensión afectiva.

Cabe señalar que este estudio presenta algunas limitaciones dado su carácter descriptivo, por tanto, las relaciones descritas no confirman causalidad. En este sentido, el estudio supone un acercamiento para entender mejor los procesos afectivos en los juegos sociomotores que desarrollan en las clases de EF.

Con respecto a la transferencia de los resultados en el campo pedagógico, se propone que al momento de programar juegos sociomotrices en la EF Primaria, el profesorado tiene que considerar que el factor de oposición o competencia motriz, puede ser muy beneficioso para el BS y la motivación intrínseca del alumnado, lo cual aportaría a la disposición con que se afronta el aprendizaje. De acuerdo a la perspectiva de género, es muy recomendable que se mantenga un equilibrio en la presencia de ambos juegos sociomotores durante las sesiones de EF. Además, sería fundamental que en la clase de EF se incluya en forma sistemática contenidos de educación emocional y moral, considerando que el factor de competencia motriz, es reconocido por producir AN en relación al BS. La toma de conciencia del profesorado con respecto al AP o al AN que pueda vivenciar el alumnado en los diferentes tipos de juegos 
sociomotrices, le permitirá considerar estos factores al momento de comunicarse con sus aprendices y poder identificarlos por medio del lenguaje oral o corporal, abordando las situaciones de acuerdo a su contexto.

\section{REFERENCIAS BIBLIOGRÁFICAS}

Abellán, J., Sáez-Gallego, N. y Reina, R. (2018). Evaluación de las actitudes hacia la discapacidad en Educación Física: Efecto diferencial del sexo, contacto previo y la percepción de habilidad y competencia. Cuadernos de Psicología del Deporte, 18(1), 133-139. Recuperado de https://revistas.um.es/cpd/article/view/282581

Alcaraz-Muñoz, V., Alonso, J. y Yuste, J. (2017). Jugar en positivo: Género y emociones en educación física. Apunts. Educación Física y Deportes, 129, 51-63. doi: 10.5672/apunts.2014-0983.es.(2017/2).129.04

Caballero, M., Alcaraz, V., Alonso, J. y Yuste, J. (2016). Intensidad emocional en la clase de educación física en función de la victoria: juegos de cooperación-oposición. Revista Electrónica Interuniversitaria de Formación del Profesora, 19(3), 123-133. doi: 10.6018/reifop.19.3.267291

Camacho, M. y Aragón, N. (2014). Ansiedad física social y educación física escolar: las chicas adolescentes en las clases de natación. Apunts. Educación Física y Deportes, 116(2), 87-94. doi: 10.5672/apunts.2014-0983.es.(2014/2).116.09

Canales, I. y Pina, I. (2014). El miedo al contacto en el voleibol. Percepciones del alumnado de Educación Física. Ágora para la Educación Física y el Deporte, 16(2), 122-136. Recuperado de http://agorarevista.blogs.uva.es/files/2014/12/agora 16 2c canales et pina.pdf

Contreras, O. y García, L. (2011). Didáctica de la Educación Física. Enseñanza de los contenidos desde el constructivismo. Madrid: Síntesis. Recuperado de https://www.libreriadeportiva.com/libro/didactica-de-la-educacion-fisica-ensenanza-de-loscontenidos-desde-el-constructivismo 39807

Conway, M. y Pleydell-Pearce, C. (2000). The construction of autobiographical memories in the self-memory system. Psychological Review, 107(2), 261-288. doi: 10.1037//0033295x.107.2.261

Duran, C., Lavega, P., Planas, A., Muñoz, R. y Pubill, G. (2014). Educación física emocional en secundaria. El papel de la sociomotricidad. Apunts. Educación Física y Deportes, 117(3), 23-32. doi: 10.5672/apunts.2014-0983.es.(2014/3).117.02

García, J. (2011). El desarrollo de la autoestima a través del área de Educación Física y el reto cooperativo. ¡Puedes!. La Peonza. Revista de Educación Física para la paz, 6, 39-50. Recuperado de https://es.scribd.com/document/94411501/peonza-ne6 
Gaviria, D. y Castejón, F. (2016). Desarrollo de valores y actitudes a través de la clase de educación física. Movimento, 22(1), 251-262. https://doi.org/10.22456/1982-8918.53455

Gil, P., y Martínez, M. (2016). Emociones auto-percibidas, por alumnos y maestros, en Educación Física en 6ํㅜ curso de primaria. Educación XX1, 19(2), 79-204. doi: 10.5944/educXX1.14230.

González, N. y Valdez, J. (2015). Validez de las escalas de afecto positivo y negativo (PANAS) en niños. Liberabit. Revista Peruana de Psicología, 21(1), 37-47. Recuperado de http://ojs3.revistaliberabit.com/publicaciones/revistas/RLE 211 validez-de-las-escalasde-afecto-positivo-y-negativo-panas-en-ninos.pdf

González-Palomares, A., Táboas-Pais, M. y Rey-Cao, A. (2017). La cultura corporal en función del género: Análisis de los libros de texto de Educación Física de Secundaria publicados durante la Ley Orgánica de Educación. Educación XX1, 20(1), 141-162. doi: 10.5944/educxx1.17506

Gutiérrez, M. y Pilsa, C. (2006). Actitudes de los alumnos hacia la Educación Física y sus profesores. Revista Internacional de Medicina y Ciencias de la Actividad Física y del Deporte, 6(24), 212-229. Recuperado de http://cdeporte.rediris.es/revista/revista24/artactitudes36.pdf

Hernández-Mendo, A. y Planchuelo, L. (2014). El incremento del desarrollo moral en las clases de Educación Física. Revista Iberoamericana de Psicología del ejercicio y el Deporte, 9(2), 369-392. Recuperado de http://www.ripedonline.com/index.php/riped/article/view/202

Lamoneda, J. y Huertas, F. (2017). Análisis de la práctica deportiva-recreativa a través de un programa de promoción en función del sexo en adolescentes españoles. Retos. Nuevas Tendencias en Educación Física, Deporte y Recreación, 32, 25-29. Recuperado de https://recyt.fecyt.es/index.php/retos/article/view/49283

Lavega, P., Planas, A. y Ruiz, P. (2014). Juegos cooperativos e inclusión en Educación Física. Revista Internacional de Medicina y Ciencias de la Actividad Física y del Deporte, 14(53), 37-51. Recuperado de http://cdeporte.rediris.es/revista/revista53/artjuegos450.htm

Lavega, P., Filella, G., Agulló, M. J., Soldevila, A. y March, J. (2011). Conocer las emociones a través de juegos: Ayuda para los futuros docentes en la toma de decisiones. Electronic Journal of Research in Educational Psychology, 9(2), 617-640. Recuperado de http://www.investigacionpsicopedagogica.org/revista/new/english/ContadorArticulo.php?519

Medina, J. (2015). Emociones según interacción motriz y su relación con la motivación hacialas áreas del currículo. EmásF. Revista Digital de Educación Física, 37, 70-81. Recuperado de http://emasf2.webcindario.com/EmasF 37.pdf 
Miralles, R., Filella, G. y Lavega, P. (2017). Educación física emocional a través del juego en educación primaria. Ayudando a los maestros a tomar decisiones. Retos. Nuevas Tendencias en Educación Física, Deporte y Recreación, 31, 88-93. Recuperado de https://recyt.fecyt.es/index.php/retos/article/view/49607

Molina, R. (2016). Los juegos cooperativos y su incidencia en los estados de ánimo y las emociones en escolares de 10-12 años. EmásF. Revista Digital de Educación Física, 41, 108-132.

Recuperado

de https://emasf.webcindario.com/Los juegos cooperativos y su incidencia en los estado $\underline{s}$ de animo y las emociones.pdf

Monforte, J. y Pérez-Samaniego, V. (2017). El miedo en educación física: Una historia reconocible. Movimento, 23(1), 85-99. doi: 10.22456/1982-8918.71272

Mujica, F., Orellana, N., Aránguiz, H. y González, H. (2016). Atribución emocional de escolares de sexto año básico en la asignatura de Educación Física y Salud. Educación

Física $y$ Ciencia, 18(2), 1-6. Recuperado de https://www.efyc.fahce.unlp.edu.ar/article/view/EFyCe015

Mujica, F., Orellana, N., y Concha, R. (2017). Emociones en la clase de Educación Física: revisión narrativa (2010-2016). Ágora para la Educación Física y el Deporte, 19(1), 119-134. doi: 10.24197/aefd.1.2017.119-134.

Mujica, F. (2018). Las emociones en la Educación Física Escolar: El aporte de la evaluación cualitativa. EmásF. Revista Digital de Educación Física, 451, 64-78. Recuperado de https://emasf.webcindario.com/Las emociones en la \%20educacion fisica escolar corr ecci\%C3\%B3n.pdf

Parlebas, P. (2001). Juegos, deporte y sociedad. Léxico de praxiología motriz. Barcelona: Paidotribo. Recuperado de http://www.paidotribo.com/ficha.aspx?cod=00567

Pellicer, I. (2011). Educación física emocional: De la teoría a la práctica. Barcelona: Inde. Recuperado de https://www.inde.com/es/productos/detail/pro id/143

Rodríguez, A. y Rocu, P. (2016). Emociones percibidas a través de las primeras experiencias de expresión corporal: estudio de casos en secundaria. EmásF. Revista digital de educación física, 40, 134-157. Recuperado de https://emasf.webcindario.com/Emociones percibidas a traves de las primeras experi encias de expresion corporal.pdf

Sandín, B. (2003). Escalas PANAS de afecto positivo y negativo para niños y adolescentes. Revista de Psicopatología y Psicología Clínica, 8(2), 173-182. doi: 10.5944/rppc.vol.8.num.2.2003.3953

Sáez de Ocáriz, U., Lavega, P., Mateu, M. y Rovira, G. (2014). Emociones positivas y educación de la convivencia escolar. Contribución de la expresión motriz cooperativa. Revista de Investigación Educativa, 32(2), 309-326. doi: 10.6018/rie.32.2.183911 
Sáez, U., Lavega, P., Lagardera, J., Costes, A. y Serna, J. (2014). ¿Por qué te peleas? Conflictos motores y emociones negativas en la clase de Educación Física: el caso de los juegos de oposición. Educatio Siglo XX1, 32(2), 71-90. doi: 10.6018/j/194091

Serna, J., Muñoz, V., Lavega, P., March-Llanes, J., Sáez de Ocáriz, U. y Hileno, R. (2017). Influencia de las tareas motrices sobres los estados de ánimo en baloncesto. Revista de Psicología del Deporte, 26(1), 37-44. Recuperado de https://www.rpdonline.com/article/view/v26-n3-serna-bardavio-munoz-etal

Spiegel, M. y Stephens, L. (2001). Estadística. México: McGraw-Hill Interamericana. Recuperado de https://www.iberlibro.com/SCHAUM-Estad\%C3\%ADstica-Terceraedici\%C3\%B3n-Murray-Spiegel/18537090226/bd

Toro, S. (2006). Conocimiento y motricidad humana, aproximaciones y desafíos. Pensamiento Educativo, 38, 62-74. $\quad$ Recuperado de http://www.pensamientoeducativo.uc.cl/files/journals/2/articles/302/public/302-707-1PB.pdf

Trigo, E. y De la Piñera, S. (2000). Manifestaciones de la motricidad. Barcelona: Inde. Recuperado de https://www.casadellibro.com/libro-manifestaciones-de-lamotricidad/9788495114075/713222

Watson, D., Clark, L. y Tellegen, A. (1988). Development and validation of brief measures of positive and negative affect: The PANAS scales. Journal of Personality and Social Psychology, 54(6), 1063-1070. doi: 10.1037//0022-3514.54.6.1063 